\title{
Relationship of Body Mass Index with Cardiorespiratory Fitness in Collegiate Females of West Bengal
}

\author{
Sandip Sankar Ghosh* \\ Assistant Professor, University of Kalyani, Kalyani, Nadia-741235, West Bengal, India
}

*Corresponding Author: Sandip Sankar Ghosh, Assistant Professor, University of Kalyani, Kalyani, Nadia-741235, West Bengal, India

\begin{abstract}
Background: Low cardiorespiratory fitness in young adults has emerged as an important factor for developing cardiovascular malfunctioning later in middle age. Increased body fatness as predicted by body mass index is an additional factor for developing cardiovascular diseases.

Objective: The objective of this study was to determine the cardiorespiratory fitness in terms of $\mathrm{VO}_{2}$ max in young healthy college females and to study the relation between body mass index and cardiorespiratory fitness.

Methodology: Thirty eight $(N=38)$ young healthy college female were selected as subjects for the study from Acharya Prafullay Chandra college, New Barackpore, Kolkata-131. The age of the subjects were ranged from 18 to 23 years. Body mass index was measured as weight (in kilograms) divided by height (in meters) squared. Cardiorespiratory fitness in terms of $\mathrm{VO}_{2}$ max was measured by Queen's College Step Test (QCT). Height and Weight were measured by Anthropometric rod and Digital Weighing machine (Tanita ${ }^{T M}$, Model: $B C$-554). In the present study for the sake of analysis of data mean and standard deviation of the variables were calculated. To test the relationship within the variables co-efficient of correlation were calculated. The level of significance was set at p<0.01 level of confidence. For statistical calculations Excel Spread Sheet of windows version 7 will be used.
\end{abstract}

Results: A significant negative correlation was observed between body mass index (BMI) and $\mathrm{VO}_{2} \mathrm{max}$ $\left(\mathrm{ml} . \mathrm{kg}^{-1} \cdot \mathrm{min}^{-1}\right)$.

Conclusion: The results suggest the striking effect of fatness on cardiorespiratory functions. Excessive amount of body fat exerts an unfavourable burden on cardiac function and oxygen uptake by working muscles. Low cardiorespiratory fitness in young adults with increased body fat could be a factor for developing cardiovascular malfunction later in middle age.

Keywords: $\mathrm{VO}_{2} \max , Q C T$, body mass index, cardiorespiratory fitness

\section{INTRODUCTION}

Cardio respiratory fitness and body composition are associated with the risk of emergence of cardiovascular diseases. Accordingly, these factors as related to health have been the focus of researchers in the field of Health and Physical Education. The incidence of cardiovascular disease is statistically and physiologically related to obesity. For example, the obese individual has a mortality rate from cardiovascular disease is $2 \frac{1}{2}$ times greater than the individual with an average or below average body weight [Fox and Browers, (1989)].

Over the past four decades, there has been an increase in the prevalence of overweight and physical fitness deterioration in adult across all genders, age and racial/ ethnic groups. It can cause many risk factors to health including coronary heart disease, forms of cancer, diabetes, hypertension, stroke, osteoarthritis respiratory problems etc. Low levels of physical activity and cardiorespiratory fitness are both associated with higher risk of all causes and disease specific mortality [Thune et. al.(1998)].

Aerobic fitness is a very good indicator of cardio respiratory fitness/cardiovascular and exercise tolerance for everyday activities which are largely aerobic for the general population [Zuluaga, (1995)]. It is considered that VO2 max or maximal aerobic capacity is only a single measure of the 
functional capacity of the oxygen system or cardio-respiratory system or the oxygen transport system [Koley, (2007)]. Research has indicated the importance of exercise in reducing the risk of cardiovascular diseases, through reduction of blood lipids, body fat, and blood pressure, improvement in myocardial function and as an independent risk factor [(Fletcher et al., 1990 and National Heart Foundation, 1991)]. Thus, the concept of fitness being defined as good condition or good health, tells us that, while we do not expect the general population to compete with athletes, an above average Vo2 max score indicates a healthy level of cardio respiratory fitness and that an individual is fit to cope with the general demands of living [(Zuluaga, 1995)]. Civilization and industrialization make our life pleasant, jubilant and luxuriant. Indeed, automation and other technologies have contributed greatly to lessening physical activities at work place and home. The assessment of cardio respiratory fitness in children and adolescent has been growing in importance because several data link the $\mathrm{VO}_{2}$ max with CVD risk factors [(Sallis, 1988 and Anderson, 1992)]. The evidence from longitudinal studies indicate that early identification of cardio vascular risk factors in children, particularly for body fat \%, blood lipids, hypertension and cardio respiratory fitness should be addressed as preventive measure in public health perspectives [(Kemper, 1989)].

A sincere attempt is made to know the research work already done in this area, the gaps and weakness of the past research, the methodology used and procedure followed from professional literature. Considering the research work in this field it can be concluded that a very little effort has been taken so far on the estimation of cardiorespiratory fitness in terms maximum oxygen consumption of female populations of West Bengal. Accordingly the present project was planned to initiate research work to evaluate cardiovascular fitness in terms of $\mathrm{VO}_{2}$ max in young healthy women of West Bengal. So, the purpose of the present study was to evaluate cardiorespiratory fitness in terms of $\mathrm{VO}_{2} \mathrm{max}$ and its relation with body mass index in young healthy college female of West Bengal.

\section{Materials AND Methods}

\subsection{Subjects}

Thirty eight $(\mathrm{N}=38)$ young healthy college female were selected as subjects for the study from Acharya Prafullay Chandra college, New Barackpore, Kolkata-131. The age of the subjects were ranged from 18 to 23 years. Those students were not included as subjects for the present study having history of cardiac disease and history of lung disease. The subjects were not any history of sports participation they were basically habituated with sedentary life style. Informed consent was taken from all the subjects. The study was approved by Institutional Ethical Committee.

\subsection{Variable Studied}

In this project two variables were studied viz.: 1) Body Mass index (BMI) 2) Cardiorespiratory fitness in terms of $\mathrm{VO}_{2} \max$.

\subsection{Measuring Procedure}

Height and Weight were measured by Anthropometric rod and Digital Weighing machine (Tanita ${ }^{\mathrm{TM}}$, Model: BC-554). Body mass index was measured as weight (in kilograms) divided by height (in meters) squared i.e.

$$
\text { B.M. I }=\frac{\text { Body mass }}{(\text { Height })^{2}}
$$

Cardiorespiratory fitness in terms of $\mathrm{VO}_{2} \max$ was measured by Queen's College Step Test (QCT). Prediction of $\mathrm{VO}_{2}$ max: Participants were asked to take complete rest for half an hour before performing the exercise so that pulmonary ventilation and pulse rate might come down to a steady state. Direct estimation of VO2max is exhaustive, laborious and difficult experimental protocol (Fox, E.L., 1973). In brief the step test was performed using a stool of 16.25 inches (or $41.30 \mathrm{~cm}$ ) height. Stepping was done for a total duration of 3 minutes at the rate of 24 cycles per minute which was set by a metronome. After completion of the exercise, the subjects were asked to remain standing comfortably and the carotid pulse rate was measured from the fifth to the twentieth second of the recovery period. This 15 second pulse rate was converted into beats per minute ( $15 \mathrm{sec}$ pulse rate $\mathrm{x} 4$ ) and the value thus obtained was put in the following equation to predict $\mathrm{VO} 2 \mathrm{max}$ in $\mathrm{ml} / \mathrm{kg} / \mathrm{min}$, as proposed by McArdle et al., 1986. 
$\mathrm{VO} 2 \max (\mathrm{ml} / \mathrm{kg} / \mathrm{min})=65.81-(0.1847 \times$ pulse rate in beats per min $)$

All experiments were performed at a room temp varying from $27-29^{\circ} \mathrm{C}$ and at a relative humidity ranging between 70 and $85 \%$.

\subsection{Statistical Analysis}

In the present study for the sake of analysis of data mean and standard deviation of the variables were calculated. To test the relationship within the variables Pearson's co-efficient of correlation were calculated. The level of significance was set at $p<0.01$ level of confidence. For statistical calculations Excel Spread Sheet of windows version 7 will be used.

\section{RESULT}

In Table-1 the mean and standard deviation, of Age (in years), Height (in meters), weight (in kilograms), Body Mass Index (BMI in $\mathrm{Kg} / \mathrm{m}^{2}$ ), QCT pulse rate (in number) and VO2 max (in ml.kg${ }^{1} \cdot \mathrm{min}^{-1}$ ) have been presented. The mean values of different parameters were presented in fig-1.

Table1: Mean and Standard Deviation of Age, Height, Weight, BMI, Pulse Rate and $\mathrm{VO}_{2}$ max of the subjects

\begin{tabular}{|l|l|l|}
\hline NAME OF THE VARIABLES & MEAN & STANDARD DEVIATION \\
\hline Age (in years) & 20.82 & 1.93 \\
\hline Height (in meters) & 1.54 & 0.56 \\
\hline Weight (in kg.) & 59.36 & 8.28 \\
\hline Body Mass Index (BMI in Kg/m ${ }^{2}$ ) & 24.97 & 4.32 \\
\hline QCT Pulse Rate (No) & 182.00 & 9.62 \\
\hline VO2 max (in ml.kg ${ }^{-1} \cdot \mathrm{min}^{-1}$ ) & 34.33 & 1.74 \\
\hline
\end{tabular}

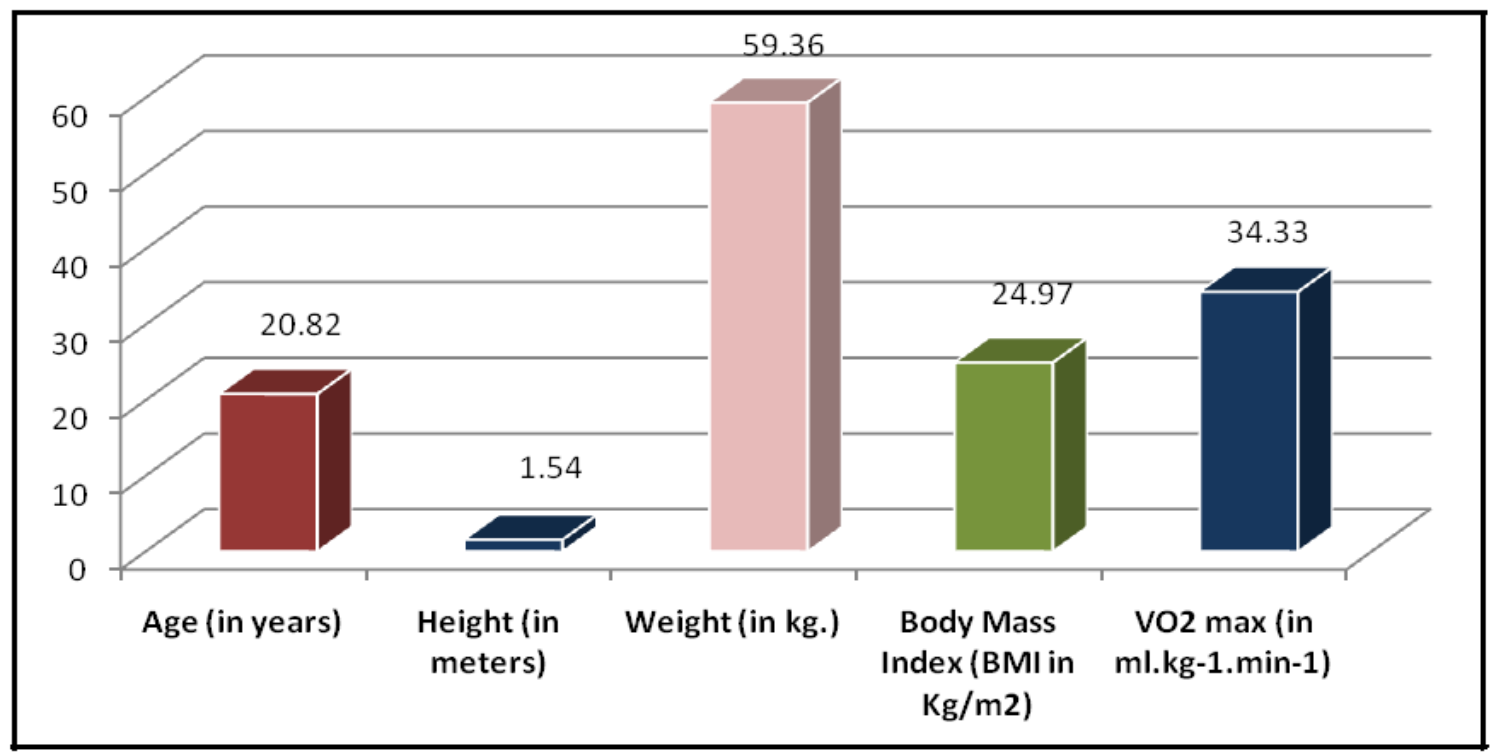

Fig1: Mean value of Age, Height, Weight, BMI, $\mathrm{VO}_{2}$ max

In table-2 the correlation between Body Mass Index and VO2 max were presented.

Table2: Pearson's Co-efficient of Correlations between BMI and VO2max

\begin{tabular}{|c|c|c|}
\hline \multicolumn{2}{|c|}{ Name of the variables Correlated } & Pearson's Co-efficient of Correlation ' $r$ ' \\
\hline BMI & $\mathrm{VO}_{2} \max$ & $-0.57 *$ \\
\hline
\end{tabular}

* At 0.01 level of significance, table value of ' $r$ ' for df (36) 0.418

From table-2 it was evident that a significant negative correlation $(-0.62$ at $\mathrm{P}<0.01)$ existed between $\mathrm{BMI}$ and $\mathrm{VO}_{2}$ max.

\section{DISCUSSION}

Results showed a significant negative correlation between BMI and cardiorespiratory fitness. This is in accordance with the previous studies which reported an inverse relation between body mass index and cardiorespiratory fitness. This suggests the striking effects of increasing BMI on cardiorespiratory function. Excessive amount of body fat exerts an unfavourable burden as well as hindering action 
towards cardiac function during exercise. Reduced cardiac function during progressive exercise results in decreased oxygen uptake. Due to deposition of proportionately high amount of fat mass, body musculature fails to uptake sufficient amount of oxygen. These findings demonstrate the importance of low cardiorespiratory fitness in young adults with increasing BMI which could be a factor for developing cardiovascular malfunctioning later in middle age.

\section{CONCLUSION}

On the basis of result \& discussion following conclusions were drawn -:

- There was a significant negative correlation between BMI and VO2max $\left(\mathrm{ml}^{\mathrm{kg}} \mathrm{kg}^{-1} \cdot \mathrm{min}^{-1}\right)$. This suggests the possibility of effect of body fat on cardiorespiratory functions.

- These findings demonstrate the importance of low cardiorespiratory fitness in young adult female with increased body fat which could be a factor for developing cardiovascular malfunctioning later in middle age.

- It is important to improve cardiorespiratory fitness in young women by engaging them in physical activities is the solution to cope up from cardiac risk.

\section{REFERENCES}

[1] Chatterjee S, Chatterjee P, Bandyopadhay A. Validity of Queen's College Step Test for use with young men. Br J Sports Med 2004;38: 289 -291.

[2] Jain AK. Cardiorespiratory response to steady state exercise in sedentary men 20 -30 years old men. Ind J Chest Dis And Allied Sci 1983;25: 172- 185.

[3] Laxmi, C.C. A study of effect of body mass index on cardiorespiratory fitness of young men, Department of physiology Sri Devaraj medical college, Tamaka, kollar, MD Dissertation 2008

[4] Astrand PO, Rodahl K. Text Book of work Physiology. 1st edition. New York: McGraw Hill; 1970.

[5] Shayamal K. Association of cardiorespiratory fitness, body composition and Blood pressure in college population of Amritsar, Punjab, India. The Internet Journal of Biological Anthropology 2007;1:1.

Citation: Sandip Sankar Ghosh. "Relationship of Body Mass Index with Cardiorespiratory Fitness in Collegiate Females of West Bengal" International Journal of Sports and Physical Education (IJSPE), vol 5, no. 3, 2019, pp. 19-22. doi: http://dx.doi.org/10.20431/2454-6380.0503004.

Copyright: (1) 2019 Authors. This is an open-access article distributed under the terms of the Creative Commons Attribution License, which permits unrestricted use, distribution, and reproduction in any medium, provided the original author and source are credited. 